\section{§23. Shape Effect of Resistance and Thermoelectric Power for BiSb in a Magnetic Field}

Hasegawa, Y. (Ion Engineering Research Institute Corp.; NEDO fellowship), Okumura, H. (Matsusaka Univ.), Yamaguchi, S.

The measurement of seebeck coefficient and magnetoresistance was done for a direction of the axis of the crystal. The magnetic field effect were observed experimentally, therefore, and we calculate the shape effect of magnetic field by two-dimensional computer code [1], which includes the Hall effect, thermoelectric effect, Nernst effect and Leduc-Righi effect for the sample.

It is shown in Figs 1, 2 that the calculation results of apparent seebeck coefficient and magnetoresistance which are considered without electrodes and with electrodes, respectively. Here, 'Without electrodes' means raw value from calculation, and 'With electrodes' from calculation corresponds to experiment. Of course, Both seebeck coefficient and magnetoresistance are equal without magnetic induction (0 Tesla). Apparent seebeck coefficient of calculation without electrodes is increasing with increasing magnetic induction till 1.5 Tesla and almost no change over 1.5 Tesla. However apparent seebeck coefficient with electrodes has a peak $\alpha\left(\mathrm{B}_{\text {peik }}\right)$, and decreasing with increasing magnetic induction over $\mathrm{B}_{\text {peak }}$. The latter tendency is good agreement with experimental result. Seebeck coefficient of calculation with electrodes and experimental result has a peak at same magnetic induction. Relative magnetoresistance from calculation without electrodes largely does not change with increasing magnetic induction and is almost constant. However, relative magnetoresistance with electrodes largely changes, and good agreement with experimental result.

A comparison between experimental result and calculation with electrodes is good agreement apparent seebeck coefficient and magnetoresistance in Figs. 1, 2. From this result, we can conclude that the electrode shape has greatly influence on the parameters of the figure of merit, in particular, magnetoresistance is varied which semiconductor is attached 'with electrodes' or 'without electrodes'. The electrode shape must be taken into account if figure of merit is estimated under the magnetic field. This electrode shape effect may be related to a difference of figure of merit, which is increasing or decreasing with increasing magnetic field. Under magnetic field, some people were stated to increase the figure of merit, and other people were stated to decrease one. To solve this paradox, we studied by means of experiment and calculation. From a comparison between experimental result to utilized single crystal Bismuth-Antimony and calculation considering phenomenological equations, their results are good agreement between 0 and 4 Tesla with concerning electrodes of semiconductor. We can conclude that seebeck coefficient and magnetoresistance have the influence of not only shape of thermoelectric semiconductor but also shape of electrode under magnetic field. Experimental results and calculation are shown that the electrode shape of thermoelectric element causes to vary parameters of figure of merit.

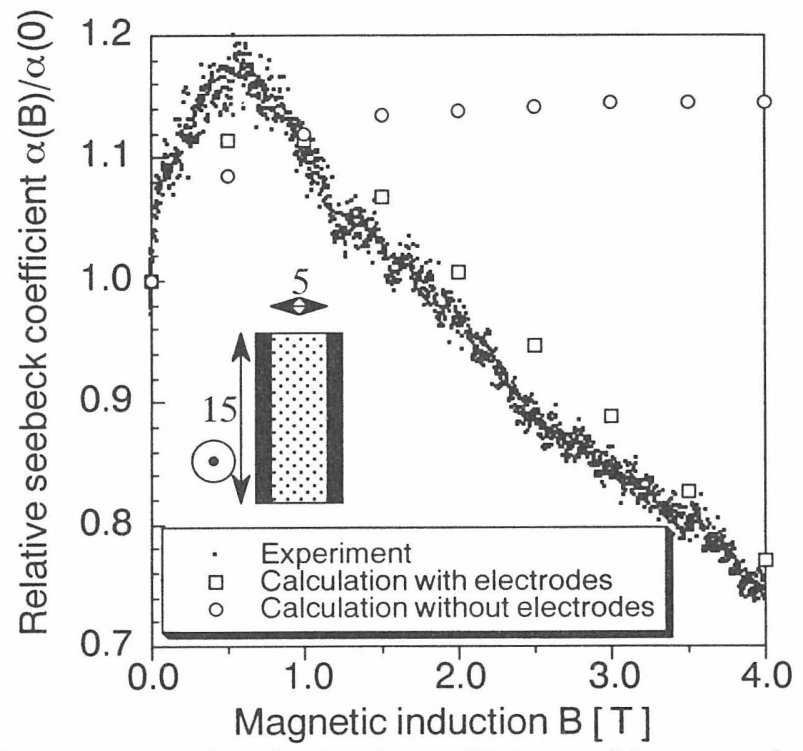

Fig. 1 Relative Seebeck coefficient with magnetic indution.

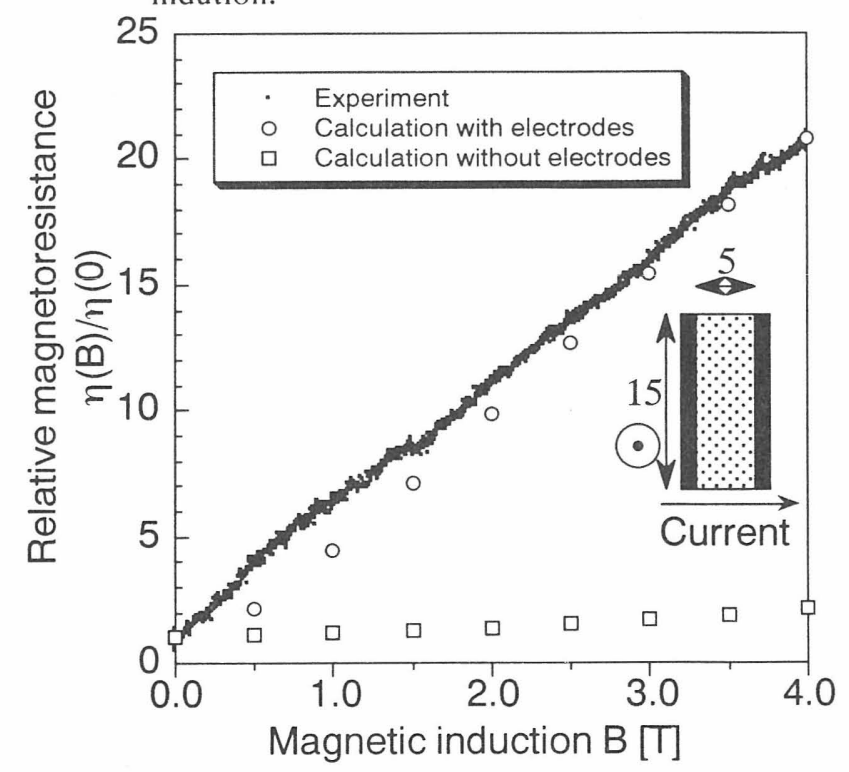

Fig. 2 Relative resistivity with magnetic induction

\section{Reference}

[1] H. Okumura, et al., 'Numerical Computation of Thermoelectric and Thermomagnetic Effects', ICT98, 